\title{
Prognostic factors for local recurrence, metastasis and survival for sebaceous carcinoma of the eyelid: observations in 100 patients
}

\author{
Ho-Seok Sa, ${ }_{1}^{1,2}$ Maria Laura Rubin, ${ }^{3}$ Shiqiong $\mathrm{Xu}_{1}{ }^{1,4}$ Jing Ning, ${ }^{3}$ Michael Tetzlaff, \\ Oded Sagiv ${ }^{1}{ }^{1}$ Thomas J Kandl, ${ }^{1}$ Bita Esmaeli ${ }^{1}$
}

\begin{abstract}
- Additional material is published online only. To view please visit the journal online (http://dx.doi.org/10.1136/ 10.1136/bjophthalmol-2018312635)
\end{abstract}

Orbital Oncology and Ophthalmic Plastic Surgery, Department of Plastic Surgery, The University of Texas MD Anderson Cancer Center, Houston, Texas, USA

${ }^{2}$ Department of Ophthalmology, Asan Medical Center, University of Ulsan College of Medicine, Seoul, South Korea

${ }^{3}$ Department of Biostatistics, The University of Texas MD Anderson Cancer Center, Houston, Texas, USA ${ }^{4}$ Department of Ophthalmology, Ninth People's Hospital, School of Medicine, Shanghai Jiao Tong University, Shanghai, China ${ }^{5}$ Department of Pathology, The University of Texas MD Anderson Cancer Center, Houston, Texas, USA

\section{Correspondence to} Dr Bita Esmaeli, Orbital Oncology and Ophthalmic Plastic Surgery, Department of Plastic Surgery, The University of Texas MD Anderson Cancer Center, Houston, TX 77030, USA; besmaeli@mdanderson. org

Received 29 May 2018 Revised 14 July 2018 Accepted 6 August 2018 Published Online First 21 August 2018

Check for updates

(c) Author(s) (or their employer(s)) 2019. No commercial re-use. See rights and permissions. Published by BMJ.

To cite: Sa H-S, Rubin ML, Xu S, et al. Br J Ophthalmol 2019:103:980-984.

\begin{abstract}
Background/aims To validate the predictive value of the American Joint Committee on Cancer (AJCC) 8thedition classification for local recurrence, metastasis and survival in patients with eyelid sebaceous carcinoma. Methods We performed a retrospective review of 100 consecutive patients with eyelid sebaceous carcinoma. Eyelid carcinomas were staged according to the AJCC 7th-edition and 8th-edition criteria. Associations between $\mathrm{T}$ and $\mathrm{N}$ categories and diseaserelated outcomes including local recurrence, lymph node metastasis, distant metastasis and survival were evaluated.

Results 60 women and 40 men had a median age of 67 years (range, 41-94 years). The proportions of patients who experienced local recurrence, lymph node metastasis, distant metastasis and death from disease were $6 \%, 21 \%, 7 \%$ and $6 \%$, respectively. Two-year and 5 -year disease-specific survival (DSS) rates were $93.8 \%$ and $92.0 \%$, respectively. There were significant correlations between (1) T2c or worse category and lymph node metastasis $(p=0.04)$ and distant metastasis $(p=0.01),(2)$ T3b or worse category and local recurrence $(p=0.01)$ and death from disease $(p=0.01)$ and $(3)$ $\mathrm{N} 1$ category at presentation and distant metastasis $(p<0.01)$ and death from disease $(p<0.01)$. The AJCC 8th-edition classification showed a better homogeneity of the T-category distribution $(p<0.01)$ and a slightly higher discrimination ability for lymph node metastasis ( $C=0.734$ vs $C=0.728$ ) than the 7 th-edition.

Conclusions T and N categories per AJCC 8thedition classification are predictive of local recurrence, metastasis and DSS outcomes for eyelid sebaceous carcinoma. Surgeons should perform strict surveillance testing for nodal and systemic metastases in patients with T2c or worse T category and/or N1 disease at presentation.
\end{abstract}

\section{INTRODUCTION}

Sebaceous carcinoma of the eyelid and ocular adnexa is a rare malignant neoplasm that can exhibit aggressive local behaviour and metastasize to regional lymph nodes and distant organs. ${ }^{1}$ The American Joint Committee on Cancer (AJCC) TNM classification for eyelid carcinoma in the 7th edition of the AJCC Cancer Staging Manual (the '7th-edition classification'), published in $2009,{ }^{2}$ was shown in previously published studies to be predictive of outcomes in patients with sebaceous carcinoma, mainly focusing on lymph node metastasis. ${ }^{3-8}$ However, the AJCC 7 th-edition classification for eyelid carcinoma sometimes led to inconsistent reporting, because the classification included subjective wording, such as 'complete tumour resection requires enucleation, exenteration or bone resection' and 'not resectable' or to artificial upstaging, because the classification automatically assigned a specific $\mathrm{T}$ category in patients with certain histopathological features, such as 'perineural invasion', regardless of the size or extent of the primary tumour. ${ }^{2}$ The 8 th-edition AJCC TNM classification for eyelid carcinoma was published in 2017 and included significant changes compared with the 7 th-edition classification. ${ }^{9}$

The purpose of this study was to determine the prognostic factors for local recurrence, metastasis and survival in patients with sebaceous carcinoma of eyelid. Specifically the predictive value of the AJCC 8th-edition TNM classification was compared with the AJCC 7th-edition criteria in terms of their ability to stratify patients with respect to these outcomes.

\section{METHODS}

The clinical records of all consecutive patients treated by one author (BE) between May 1999 and November 2017 for histologically proven eyelid sebaceous carcinoma were reviewed for age, sex, race/ethnicity, previous medical history, clinical information needed for tumour classification at presentation (tumour location, clinical tumour size, involvement of the eyelid margin or not and presence and location of lymph node and/or distant metastasis), treatment information (surgical treatments, sentinel lymph node (SLN) biopsy, adjuvant radiotherapy and topical chemotherapy) and outcomes of interest (local recurrence, lymph node metastasis, distant metastasis, death from disease and death from any cause). Histopathological records were also reviewed for tumour size based on the surgical specimen, involvement of the eyelid margin or not and presence of intraepithelial neoplasia, perineural invasion and lymphovascular invasion. Each tumour was classified when the patient sought treatment at our centre according to both the AJCC 8th-edition criteria for eyelid carcinoma (see online supplementary table 1 ) and the AJCC 7th-edition criteria. ${ }^{2}$

This retrospective study carried out following the tenets of the Declaration of Helsinki. 


\section{Statistical considerations}

Time to outcomes of interest was measured from the date of surgery, and patients who did not experience the event were censored at the date of last contact or death. Survival curves were estimated using the Kaplan-Meier method, and differences in survival among groups were assessed using 2-sided log-rank tests. Associations between the $\mathrm{T}$ category and time-to-event outcomes diagnosed during follow-up were tested by log-rank tests. Associations between the T category and outcomes diagnosed at presentation and by the last contact were assessed using Fisher's exact test. Distributions of patients among the different TNM designations per the AJCC 7th-edition and 8th-edition criteria were compared using a $\chi^{2}$ test for homogeneity, and the Harrell's C-statistic was used to compare the discrimination abilities of two different criteria. Statistical analyses were conducted in R, V.3.4.2.

\section{RESULTS}

A total of 121 patients diagnosed with eyelid sebaceous carcinoma were treated during the study period; only 100 patients were included in the study; the rest were seen for second opinions only or otherwise did not have adequate follow-up data. Patient, tumour and treatment characteristics are summarised in table 1 . Sixty women and 40 men had a median age of 67 years, and the median and mean follow-up were 31.5 and 39.2 months, respectively. Sixteen (16\%) patients had recurrent tumours at presentation. One patient had Muir-Torre syndrome. The AJCC 8th-edition TNM designations at presentation were as follows: T1aN0M0, 3 patients; T1bN0M0, 24; T1cN0M0, 6; T2bN0M0, 13; T2cN0M0, 7; T2cN1M0, 1; T3bN0M0, 10; T3cN0M0, 21; T3cN1M0, 5; T4aN0M0, 6; T4aN1M0, 1 and T4bN1M0, 3 .

All patients underwent imaging study and ultrasonography of the regional lymph nodes ( \pm fine-needle aspiration) at baseline. Thirty patients underwent SLN biopsy, and five of them had positive nodes. Ninety-nine (99\%) patients had surgery as the initial treatment, and the other patient (T4bN1M0) underwent chemoradiation because he refused exenteration of the only eye. Eight of 99 patients who had surgery had positive final margins for carcinoma in situ; the rest had final negative margins. Positive margins were left behind so as to avoid the need for an orbital exenteration, and only adjuvant topical chemotherapy was administered. Fourteen patients had an orbital exenteration. Histopathological records showed that 31 tumours had evidence of 'pagetoid' intraepithelial neoplasia (table 1).

Outcomes by $\mathrm{T}$ category are summarised in table 2 . Four of the six patients with local recurrence had intraepithelial neoplasia and a final resection margin positive for carcinoma in situ. By last contact, the total number of patients who experienced local recurrence, lymph node metastasis, distant metastasis and death from disease was 6, 21, 7 and 6, respectively. All six patients who died from disease had distant metastasis, and five of those patients also had lymph node metastasis. Final status was as follows: no evidence of disease, 85 patients; alive with disease, 3 ; dead of disease, 6 and dead of unrelated causes, 6 .

\section{Validation of the AJCC 8th-edition T and N categories}

All six patients with local recurrence during follow-up had T3b or worse category (table 2). Time to local recurrence was significantly longer for patients with T1/T2 than for patients with T3/T4 disease $(p=0.01$; table 3$)$. Patients with T3b or worse category had a higher risk of local recurrence than patients with less than $\mathrm{T} 3 \mathrm{~b}$ disease $(\mathrm{p}=0.01)$. Intraepithelial neoplasia

\begin{tabular}{|c|c|}
\hline Characteristic & Value \\
\hline Age, median (min, max), years & $67(41,94)$ \\
\hline \multicolumn{2}{|l|}{ Sex, No. $(\%)$} \\
\hline Male & $40(40)$ \\
\hline Female & $60(60)$ \\
\hline \multicolumn{2}{|l|}{ Race/ethnicity, №. (\%) } \\
\hline White & $71(71)$ \\
\hline Hispanic & $19(19)$ \\
\hline Black & $4(4)$ \\
\hline Asian & $6(6)$ \\
\hline \multicolumn{2}{|l|}{ Laterality, №. (\%) } \\
\hline Right & $46(46)$ \\
\hline Left & $54(54)$ \\
\hline \multicolumn{2}{|l|}{ Location, No. (\%) } \\
\hline Upper lid & $53(53)$ \\
\hline Lower lid & $26(26)$ \\
\hline Both lids & $18(18)$ \\
\hline Medial canthus & $3(3)$ \\
\hline \multicolumn{2}{|l|}{ Intraepithelial neoplasia, №. (\%) } \\
\hline No & $69(69)$ \\
\hline Yes & $31(31)$ \\
\hline \multicolumn{2}{|l|}{ Perineural invasion, No. (\%) } \\
\hline No & $93(93)$ \\
\hline Yes & $7(7)$ \\
\hline \multicolumn{2}{|l|}{ Lymphovascular invasion, №. (\%) } \\
\hline No & $98(98)$ \\
\hline Yes & $2(2)$ \\
\hline \multicolumn{2}{|l|}{ Initial treatment, №. (\%) } \\
\hline Local excision with reconstruction & $85(85)$ \\
\hline Exenteration & $14(14)$ \\
\hline Chemoradiation therapy & $1(1)$ \\
\hline \multicolumn{2}{|l|}{ Adjuvant treatment, №. (\%) } \\
\hline None & $83(83)$ \\
\hline Radiation therapy & $9(9)$ \\
\hline Chemoradiation therapy & $2(2)$ \\
\hline Topical chemotherapy with mitomycin C & $6(6)$ \\
\hline
\end{tabular}

was significantly associated with the risk of local recurrence $(p=0.02)$, but perineural invasion did not correlate with local recurrence $(p=0.36)$.

For new lymph node metastasis found during follow-up, the T category was significantly associated with the risk of lymph node metastasis $(p=0.03)$. Patients with T3/T4 disease had significantly shorter time to lymph node metastasis than did patients with T1/T2 disease $(p=0.01$; table 3$)$. Patients with T2c or worse category had a higher risk of lymph node metastasis than patients with less than $T 2 c$ disease $(p=0.04$; figure $1 \mathrm{~A})$.

Of the seven patients with distant metastasis, six had T3 or $\mathrm{T} 4$ disease and one had $\mathrm{T} 2 \mathrm{c}$ disease. $\mathrm{T}$ category was significantly associated with the risk of distant metastasis $(p<0.01)$. Patients with T2c or worse category had a higher risk of distant metastasis than patients with less than T2c disease $(p=0.01$; figure 1B). Patients with lymph node metastasis at presentation (N1) had a higher risk of distant metastasis than patients with N0 disease ( $<<0.01$; figure $2 \mathrm{~A})$.

In the entire series, the 2-year disease-specific survival (DSS) rate was $93.8 \%$ (95\% CI $88.1 \%$ to $99.9 \%)$, and the 5 -year DSS rate was $92.0 \%$ (95\% CI $85.6 \%$ to $99 \%)$. All six patients who died from disease had T3b or worse category, and all died from 
Table 2 Distribution of outcomes by T category $(\mathrm{N}=100)$

\begin{tabular}{|c|c|c|c|c|c|c|}
\hline \multirow{2}{*}{ T category } & \multirow{2}{*}{$\begin{array}{l}\text { Local recurrence } \\
\text { during follow-up }\end{array}$} & \multicolumn{3}{|c|}{ Lymph node metastasis } & \multirow{2}{*}{$\begin{array}{l}\text { Distant metastasis } \\
\text { during follow-up }\end{array}$} & \multirow{2}{*}{$\begin{array}{l}\text { Death from diseas } \\
\text { during follow-up }\end{array}$} \\
\hline & & At presentation & During follow-up & By last contact & & \\
\hline $\mathrm{T} 1(\mathrm{n}=33)$ & 0 & 0 & 1 & 1 & 0 & 0 \\
\hline $\mathrm{T} 2(\mathrm{n}=21)$ & 0 & 1 & 1 & 2 & 1 & 0 \\
\hline T3 $(n=36)$ & 5 & 5 & 7 & 12 & 1 & 1 \\
\hline T4 $(n=10)$ & 1 & 4 & 3 & 6 & 5 & 5 \\
\hline Total & 6 & 10 & $12^{*}$ & 21 & 7 & $6 \dagger$ \\
\hline
\end{tabular}

*Eleven of these 12 patients had no lymph node metastasis at presentation; 1 patient had lymph node metastasis at presentation and had an additional lymph node metastasis documented during follow-up.

${ }^{\dagger}$ All six patients who died from disease had distant metastasis, and five of those patients also had lymph node metastasis.

distant metastasis. DSS was significantly worse for patients with $\mathrm{T} 3 \mathrm{~b}$ or worse category than for patients with less category $(\mathrm{p}=0.01)$. The median DSS time for T4 disease was 27 months, which was worse than DSS time for T3 disease $(\mathrm{p}<0.01$; table 3 , figure 1C). Patients with lymph node metastasis at presentation (N1) had a worse DSS than patients with N0 disease $(\mathrm{p}<0.01$; figure $2 \mathrm{~B})$. An additional six patients died from unrelated causes. Overall survival (OS) was significantly different among the T1, T3 and T4 groups $(\mathrm{p}<0.01)$.

There were no significant differences between patients with and without recurrent eyelid carcinoma at presentation with respect to local recurrence-free survival $(\mathrm{p}=0.91)$, lymph node metastasis-free survival $(\mathrm{p}=0.62)$, distant metastasis-free survival $(p=0.48)$, DSS $(p=0.61)$ or OS $(p=0.46)$.

\section{Comparison between AJCC 8th-edition and 7th-edition classifications}

The 100 patients were stratified at presentation into only 8 different TNM categories according to the AJCC 7th-edition criteria but 12 categories according to the 8 th-edition. The distribution of T1:T2:T3:T4 tumours according to the AJCC 7th-edition and 8th-edition criteria was 1:52:47:0 and 33:21:36:10, respectively, which were significantly different from each other $(\mathrm{p}<0.01)$. These findings suggest that the AJCC 8th-edition criteria offered a more precise and homogeneous categorisation of tumours than did the 7 th-edition criteria.

Whether the 7 th-edition or 8th-edition classification was applied, patients with T3/T4 disease had a significantly higher risk of lymph node metastasis and worse OS than patients with $\mathrm{T} 1 / \mathrm{T} 2$ disease in univariate Cox models $(\mathrm{p}<0.05$ for each classification). However, the model of the AJCC 8th-edition classification had slightly higher discrimination ability for lymph node metastasis $(C=0.734$ vs $C=0.728)$ and $O S(C=0.702$ vs $\mathrm{C}=0.693$ ) than the model of the 7 th-edition. Owing to the limited number of events, the discrimination ability for the rest outcomes could not be compared.

\section{DISCUSSION}

The major finding in this large single-centre cohort study is that the $\mathrm{T}$ and $\mathrm{N}$ categories according to the AJCC 8th-edition classification have prognostic value for outcomes in patients with eyelid sebaceous carcinoma. We found a significant correlation between T2c or worse category and lymph node metastasis and distant metastasis. We also found that T3b or worse category significantly correlated with local recurrence and death from disease. Put another way and in more practical clinical terms, our findings suggest that an eyelid sebaceous carcinoma of approximately $>20 \mathrm{~mm}$ in greatest dimension correlates with increased risk for local recurrence, lymph node metastasis, distant metastasis and death from disease. When a tumour involves the full thickness of the eyelid, the risk for lymph node metastasis and distant metastasis is increased even for tumours of $>10 \mathrm{~mm}$.

We also found a significant correlation between lymph node metastasis (N1 disease) at presentation and distant metastasis and death from disease. Furthermore, we found that the AJCC 8 th-edition classification was superior to the 7 th-edition classification in terms of stratifying patients with respect to primary tumour characteristics.

The overall rates of local recurrence, lymph node metastasis, distant metastasis and death from disease in this series-6\%, $21 \%, 7 \%$ and $6 \%$, respectively-are in line with previously reported rates, which range from $5 \%$ to $18 \%, 8 \%$ to $23 \%, 2 \%$ to $14 \%$ and $2 \%$ to $10 \%$, respectively. ${ }^{13-8} 10$

Previous studies using the AJCC 7th-edition classification have validated that $\mathrm{T}$ category is the main prognostic factor in patients with eyelid sebaceous carcinoma, particularly for the risk of lymph node metastasis. ${ }^{3-8}$ Our report published in $2012^{3}$ based on a mostly Caucasian cohort and additional studies that

Table 3 Log-rank test $p$ values* comparing T categories pairwise for each outcome

\begin{tabular}{lllllr}
\hline & \multicolumn{4}{l}{ Time to outcomes of interest } \\
\cline { 2 - 6 } T category & Local recurrence & Lymph node metastasist & Distant metastasis & Death from disease & Death from any cause \\
\hline T1 vs T2 $\neq$ & - & 0.23 & 0.21 & - & 0.31 \\
T1 vs T3 & 0.02 & 0.03 & 0.32 & 0.31 & 0.28 \\
T1 vs T4 & 0.05 & $<0.01$ & $<0.01$ & $<0.01$ & 0.01 \\
T2 vs T3 & 0.10 & 0.11 & 0.77 & 0.45 & $<.18$ \\
T2 vs T4 & 0.11 & 0.10 & $<0.01$ & $<0.01$ & 0.01 \\
T3 vs T4 & 0.9 & 0.45 & $<0.01$ & $<0.01$ & 0.01 \\
T1/T2 vs T3/T4 & 0.01 & 0.01 & 0.01 & 0.01 & \\
\hline
\end{tabular}

${ }^{*} P$ values are not adjusted for multiple comparisons owing to the exploratory nature of the study.

tLymph node metastasis during follow-up (not at presentation) was the subject of analysis.

$\ddagger$ Neither local recurrence nor death from disease occurred in patients with $\mathrm{T} 1$ and $\mathrm{T} 2$ disease; $\mathrm{p}$ values cannot be computed for these outcomes. 

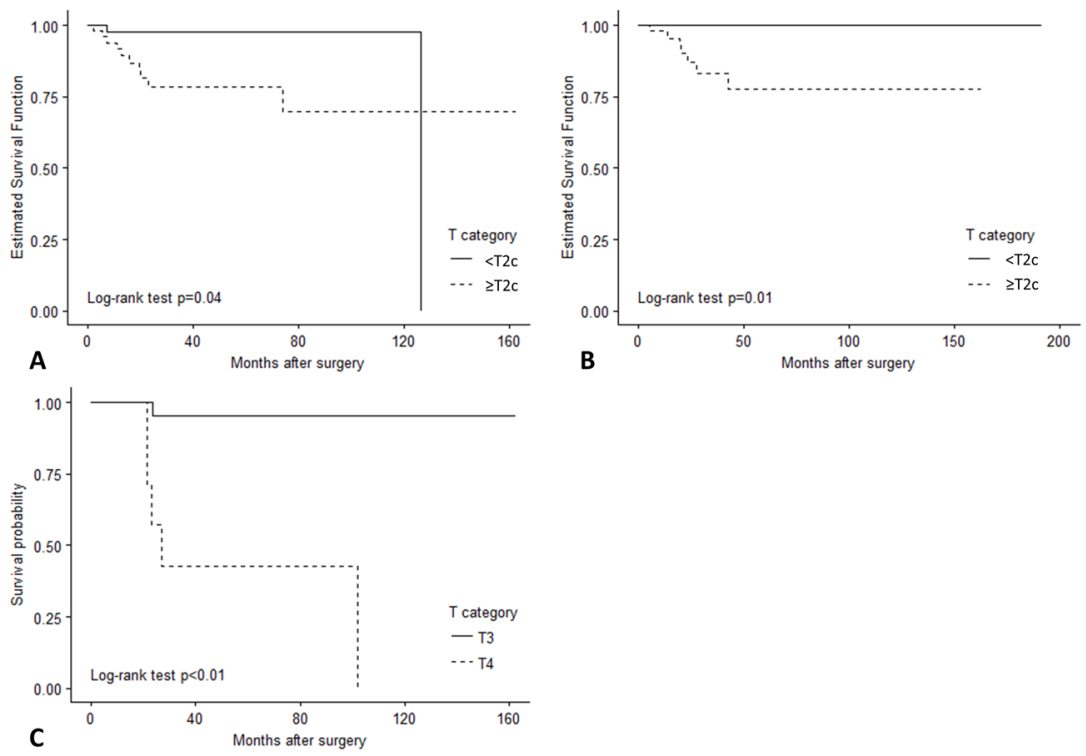

Figure 1 Kaplan-Meier curves for time to outcomes of interest by $\mathrm{T}$ category at presentation (A) lymph node metastasis during follow-up ( $<\mathrm{T} 2 \mathrm{c}$ vs $\geq \mathrm{T} 2 \mathrm{c}$ ), (B) distant metastasis ( $<\mathrm{T} 2 \mathrm{c}$ vs $\geq \mathrm{T} 2 \mathrm{c}$ ) and (C) death from disease by $\mathrm{T}$ category.

followed in Korean, ${ }^{4}$ and Chinese ${ }^{7}$ populations suggested that 7 th-edition $\mathrm{T} 2 \mathrm{~b}$ (tumour $>10 \mathrm{~mm}$ or involving the full thickness of the eyelid) or worse category is significantly associated with lymph node metastasis. In the current report of 100 mostly Caucasian patients, we found that 8th-edition T2c (tumour $>10 \mathrm{~mm}$ and involving the full thickness of the eyelid) or worse category is significantly associated with higher risk of lymph node and distant metastasis. The definitions of $\mathrm{T}$ categories in the 8th-edition classification have been modified and refined compared with the 7 th-edition as will be discussed in the subsequent paragraphs.

The current series revealed that AJCC 8th-edition T category is also associated with other important outcomes, including local recurrence. We found that patients in the combined T3 $(>20$ $\mathrm{mm}$ )/T4 category had worse local recurrence-free survival than patients in the combined T1/T2 category. The overall local recurrence rate was only $6 \%$ and there was a significant correlation between pagetoid intraepithelial neoplasia and risk of local recurrence $(p=0.02)$. This is in contrast to findings in some previous smaller case series which showed a borderline correlation ${ }^{10}$ or no correlation. ${ }^{11}$ Topical chemotherapy as adjuvant treatment ${ }^{1213}$ may be beneficial for local control of disease in patients with intraepithelial neoplasia. Although intraepithelial neoplasia is not a criterion for determining AJCC T category, it should be recorded as a prognostic factor for eyelid sebaceous carcinoma.

Lymph node metastasis has been clinically accepted as an important prognostic factor in many head and neck carcinomas. However, the ophthalmic literature is lacking studies on outcomes for eyelid sebaceous carcinoma according to the AJCC $\mathrm{N}$ category. The current series revealed that $\mathrm{N} 1$ disease at presentation was associated with increased risk of distant metastasis and death from disease. It has been debated whether lymph node metastasis simply reflects tumour aggressiveness or is a potential source of further distant metastasis, ${ }^{14}$ but there is evidence that primary tumours with lymph node metastasis more commonly spread to distant sites. ${ }^{14}$ SLN biopsy for eyelid carcinoma, which has been recommended because of its value for staging and thus impact on treatment strategy, is also based on the assumption that lymphatic spread occurs before vascular spread. ${ }^{15}$ The findings of our current study demonstrate the value of a thorough lymph node workup at presentation, including SLN biopsy and close nodal surveillance during follow-up. At our institution, we have been enrolling patients in a prospective clinical trial evaluating SLN biopsy for eyelid sebaceous carcinoma for over a decade, and the current cohort showed a reasonable yield of about 15\% (5 positive SLNs/30 patients). Our selection criteria have evolved on the basis of the findings reported herein, and
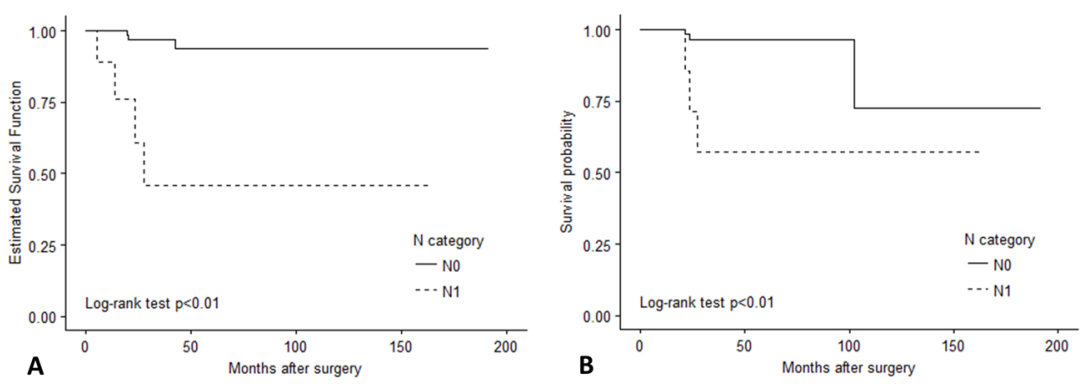

Figure 2 Kaplan-Meier curves for time to outcomes of interest by N category at presentation (A) distant metastasis (N0 vs N1) and (B) death from disease (N0 vs N1). 
we currently offer SLN biopsy to patients with eyelid sebaceous carcinoma only if the tumour is larger than $10 \mathrm{~mm}$.

The current report also showed that the new AJCC 8th-edition classification for eyelid carcinoma is better than the 7th-edition classification for stratifying patients in terms of primary tumour features and outcomes. The AJCC 8th-edition classification ${ }^{9}$ has some notable differences compared with the 7 th-edition. $^{2}$ For instance, in the 8th-edition classification, T1, T2 and T3 are now determined only by tumour size and involvement of the eyelid margin or not, and the standard sizes of tumours have been refined. The 8 th-edition classification also no longer has subjective wording included in the 7th-edition classification. These changes should reduce interobserver variability and less artificial upstaging. We found that the AJCC 8th-edition classification showed a more homogeneous distribution across $\mathrm{T}$ categories and higher discrimination ability regarding lymph node metastasis and OS than the AJCC 7th-edition.

This study had a few limitations. First, this study was based on data retrospectively reviewed from a tertiary cancer centre in the USA. There is potential for error in collecting data as complete tumour information was not always available in patients referred after previous partial surgeries or other treatments. However, we excluded all patients for whom we could not find reliable data. The patients in our series may have a bias towards more advanced or recurrent cases. The majority of patients were white, and application of our results to other ethnic populations may be limited. Second, multivariable and adjusted analyses were not performed owing to the limited number of metastatic events. However, the current series with 100 patients represents one of the largest single-centre cohorts of patients with eyelid sebaceous carcinoma, and all patients were evaluated and managed with a uniform method by one practitioner and with standardised methods of staging and follow-up surveillance; this is a major strength of the data presented in this report. Although all patients with a diagnosis of sebaceous carcinoma of the eyelid are followed at our centre for 5 years after definitive treatments, we acknowledge that at the time of preparation of this report the median and mean follow-up time was less than this (at 31.5 and 39.2 months, respectively). However, it is important to note that most cases of metastasis and local recurrence (major endpoints of this report) occur within the first 2 years after definitive treatment for sebaceous carcinoma and an occasional one might occur within the first 3 years and it would be quite a rare event to have metastasis beyond 5 years. Given these insights, the authors believe that the follow-up time for this cohort is adequate to draw the conclusions of the paper.

To the best of our knowledge, this study is the first case series of eyelid sebaceous carcinoma staged using the AJCC 8th-edition TNM classification; thus, the first validation report of the newly published 8th-edition criteria that went into effect as of January 2018. The AJCC 8th-edition classification for eyelid carcinoma provides a more homogenous T-category distribution than the 7th-edition classification, and $\mathrm{T}$ and $\mathrm{N}$ categories at presentation significantly correlated with the most important outcomes for eyelid sebaceous carcinoma. Our findings suggest that surgeons should perform strict surveillance testing for regional nodal and systemic metastases in patients with eyelid sebaceous carcinoma with $\mathrm{T} 2 \mathrm{c}$ or worse $\mathrm{T}$ category and/or N1 disease at presentation.

Contributors Conception and design: $\mathrm{BE}, \mathrm{H}-\mathrm{SS}$. Analysis and interpretation of data: MLR, JN, H-SS and BE. Drafting the article or revising it critically for important intellectual content: all authors. Final approval of the version to be published: BE.

Funding The authors have not declared a specific grant for this research from any funding agency in the public, commercial or not-for-profit sectors.

Competing interests None declared.

Patient consent Not required.

Ethics approval Institutional Review Board of the Ethics Committee at The University of Texas MD Anderson Cancer Center, Houston, Texas, USA

Provenance and peer review Not commissioned; externally peer reviewed.

\section{REFERENCES}

1 Shields JA, Demirci H, Marr BP, et al. Sebaceous carcinoma of the eyelids: personal experience with 60 cases. Ophthalmology 2004;111:2151-7.

2 Ainbinder DJ, Esmaeli B, Groo SC, et al. Introduction of the 7th edition eyelid carcinoma classification system from the American Joint Committee on CancerInternational Union Against Cancer staging manual. Arch Pathol Lab Med 2009;133:1256-61.

3 Esmaeli B, Nasser QJ, Cruz H, et al. American Joint Committee on Cancer T category for eyelid sebaceous carcinoma correlates with nodal metastasis and survival. Ophthalmology 2012;119:1078-82.

4 Choi YJ, Jin HC, Lee MJ, et al. Prognostic value of clinical and pathologic T stages defined by the American Joint Committee on Cancer for eyelid sebaceous carcinoma in Korea. Jpn J Ophthalmol 2014;58:327-33.

5 Kaliki S, Gupta A, Ali MH, et al. Prognosis of eyelid sebaceous gland carcinoma based on the tumor (T) category of the American Joint Committee on Cancer (AJCC) classification. Int Ophthalmol 2016:36:681-90.

6 Ford J, Thakar S, Thuro B, et al. Prognostic value of the staging system for eyelid tumors in the 7th edition of the American Joint Committee on Cancer Staging Manual. Ophthalmic Plast Reconstr Surg 2017;33:317-24

7 Lam SC, Li EYM, Yuen HKL. 14-year case series of eyelid sebaceous gland carcinoma in Chinese patients and review of management. Br J Ophthalmol 2018:bjophthalmol-2017-311533.

8 Watanabe A, Sun MT, Pirbhai A, et al. Sebaceous carcinoma in Japanese patients: clinical presentation, staging and outcomes. Br J Ophthalmol 2013;97:1459-63.

9 Esmaeli B, Dutton JJ, Graue GF. Ophthalmic sites: carcinoma of the eyelid. In: Amin MB ES, Greene FL, Byrd DR, eds. AJCC Cancer Staging Manual. 8th ed.. New York: Springer, 2017:779-86.

10 Takahashi Y, Takahashi E, Nakakura S, et al. Risk factors for local recurrence or metastasis of eyelid sebaceous gland carcinoma after wide excision with paraffin section control. Am J Ophthalmol 2016;171:67-74.

11 Chao AN, Shields CL, Krema H, et al. Outcome of patients with periocular sebaceous gland carcinoma with and without conjunctival intraepithelial invasion. Ophthalmology 2001;108:1877-83.

12 Kaliki S, Ayyar A, Dave TV, et al. Sebaceous gland carcinoma of the eyelid: clinicopathological features and outcome in Asian Indians. Eye 2015;29:958-63.

13 Shields CL, Naseripour M, Shields JA, et al. Topical mitomycin-C for pagetoid invasion of the conjunctiva by eyelid sebaceous gland carcinoma. Ophthalmology 2002;109:2129-33

14 Cho JK, Hyun SH, Choi N, et al. Significance of lymph node metastasis in cancer dissemination of head and neck cancer. Trans/ Oncol 2015;8:119-25.

15 Pfeiffer ML, Savar A, Esmaeli B. Sentinel lymph node biopsy for eyelid and conjunctival tumors: what have we learned in the past decade? Ophthalmic Plast Reconstr Surg 2013;29:57-62. 